Proceedings of the Institute of Mathematics and Mechanics,

National Academy of Sciences of Azerbaijan

Volume 46, Number 1, 2020, Pages 129-143

https://doi.org/10.29228/proc.23

\title{
MAXIMAL AND POTENTIAL OPERATORS ASSOCIATED WITH GEGENBAUER DIFFERENTIAL OPERATOR ON GENERALIZED MORREY SPACES
}

\author{
ELMAN J. IBRAHIMOV, SAADAT A. JAFAROVA, AND S. ELIFNUR EKINCIOGLU
}

\begin{abstract}
In this paper we study the boundedness of the maximal $(G-$ maximal) and potential ( $G$-potential) operators associated with Gegenbauer differential operator on generalized $G$-Morrey spaces. The results of this paper are generalizations of the corresponding results to generalized $G$-Morrey spaces and modified Morrey spaces. We obtain also analogs of E.Nakai's results for the Hardy-Littlewood maximal operator and the Riesz potential in generalized Morrey spaces.
\end{abstract}

\section{Introduction}

In 2011, in the paper [11] new integral transformations that formed the basis of theory of Harmonic analysis of the Gegenbauer differential operator were constructed. Later, this theory was intensively developed in various directions: approximation theory, imbedding theory, transformation theory, theory of maximal functions and potential theory (see [4-8, 9-11]). The basis of this theory was the Gegenbauer differential operator $G$ (see [1]). In [1], various representations (through integral and hypergeometrical functions) of eigen-functions of this operator, relations between them, formulas of addition and product for these functions, asymptotic formulas, etc are given. The reader can find detailed information in the mentioned paper [1].

One of the important directions of the Gegenbauer harmonic analysis is the boundedness of maximal operator and potential generated by the Gegenbauer differential operator $G$.

The boundedness of the maximal ( $G$-maximal) and potential $(G$-potential) operators associated with Gegenbauer differential operator $G$.

$$
G \equiv G_{\lambda}=\left(x^{2}-1\right)^{\frac{1}{2}-\lambda} \frac{d}{d x}\left(x^{2}-1\right)^{\lambda+\frac{1}{2}} \frac{d}{d x}, x \in(1, \infty), \lambda \in\left(0, \frac{1}{2}\right)
$$

on the Lebesgue, Morrey and modified Morrey spaces is considered in $[3,4,5]$.

In the present paper, we introduce a generalized Gegenbauer-Morrey ( $G$-Morrey) space $\mathcal{M}_{p, \lambda, \omega}\left(\mathbb{R}_{+}, G\right)$, and estimate $G$-maximal and $G$ - potential operators generated by Gegenbauer differential operator $G$. The obtained result is an analog of

2010 Mathematics Subject Classification. 42B20, 42B25, 42B35.

Key words and phrases. $G$-maximal operator, $G$-potential, generalized $G$-Morrey space. 
the corresponding theorems obtained for the Hardy-Littlewood maximal operator and the Riesz potential in [16].

\section{Definition and notation}

Let $H(x, r)=(x-r, x+r) \cap(0, \infty), r \in(0, \infty), x \in(0, \infty)=\mathbb{R}_{+}$. For all measurable sets $E \subset(0, \infty)$, put $\mu E=|E|_{\lambda}=\int_{E} s h^{2 \lambda} t d t$.

For $1 \leq p \leq \infty$ let $L_{p, \lambda}\left(\mathbb{R}_{+}, G\right)$ be the space of functions measurable on $\mathbb{R}_{+}$ with the finite norm

$$
\begin{gathered}
\|f\|_{L_{p, \lambda}}=\left(\int_{0}^{\infty}|f(\operatorname{cht})|^{p} s h^{2 \lambda} t d t\right)^{\frac{1}{p}}, 1 \leq p<\infty \\
\|f\|_{\infty, \lambda} \equiv\|f\|_{\infty}=\underset{t \in(0, \infty)}{\operatorname{ess} \sup }|f(\operatorname{cht} t)|, p=\infty
\end{gathered}
$$

In [5], the following notation is introduced.

Let $1 \leq p<\infty, 0<\lambda<\frac{1}{2}, 0 \leq \gamma \leq 2 \lambda+1,[r]_{1}=\min \{1, r\}$. We denote by $L_{p, \lambda, \gamma}\left(\mathbb{R}_{+}, G\right), \mathbb{R}_{+}=(0, \infty)$, the $G$-Morrey space, and by $\widetilde{L}_{p, \lambda, \gamma}\left(\mathbb{R}_{+}, G\right)$ the modified $G$-Morrey space, as the set of locally integrable functions $f(\operatorname{ch} x), x \in$ $\mathbb{R}_{+}$, with the finite norms

$$
\begin{aligned}
& \|f\|_{L_{p, \lambda, \gamma}}=\sup _{x, r>0}\left(r^{-\gamma} \int_{H(x, r)}|f(c h t)|^{p} s h^{2 \lambda} t d t\right)^{\frac{1}{p}} \\
& \|f\|_{\widetilde{L}_{p, \lambda, \gamma}}=\sup _{x, r>0}\left([r]_{1}^{-\gamma} \int_{H(x, r)}|f(c h t)|^{p} s h^{2 \lambda} t d t\right)^{\frac{1}{p}},
\end{aligned}
$$

respectively.

Note that $\widetilde{L}_{p, \lambda, 0}\left(\mathbb{R}_{+}, G\right)=L_{p, \lambda, 0}\left(\mathbb{R}_{+}, G\right)=L_{p, \lambda}\left(\mathbb{R}_{+}, G\right)$.

If $1 \leq p<\infty, 0<\lambda<\frac{1}{2}, 0 \leq \gamma \leq 2 \lambda+1$, then

$$
\widetilde{L}_{p, \lambda, \gamma}\left(\mathbb{R}_{+}, G\right)=L_{p, \lambda, \gamma}\left(\mathbb{R}_{+}, G\right) \cap L_{p, \lambda}\left(\mathbb{R}_{+}, G\right)
$$

and

(see [5], Lemma 2.2).

$$
\|f\|_{\widetilde{L}_{p, \lambda, \gamma}}=\max \left\{\|f\|_{L_{p, \lambda, \gamma},\|f\|_{L_{p, \lambda}}}\right\}
$$

If $\gamma<0$ or $\gamma>2 \lambda+1$, then $L_{p, \lambda, \gamma}\left(\mathbb{R}_{+}, G\right)=\widetilde{L}_{p, \lambda, \gamma}\left(\mathbb{R}_{+}, G\right)=\Theta$, where $\theta$ is the set of all functions equivalent to 0 on $\mathbb{R}_{+}$.

Let $1 \leq p<\infty, 0<\lambda<\frac{1}{2}, 0 \leq \gamma \leq 2 \lambda+1$. We denote by $W L_{p, \lambda, \gamma}\left(\mathbb{R}_{+}, G\right)$ the weak $G$-Morrey space, and $W \widetilde{L}_{p, \lambda, \gamma}\left(\mathbb{R}_{+}, G\right)$ the modified weak $G$-Morrey space as the set of locally integrable functions $f(\operatorname{ch} x), x \in \mathbb{R}_{+}$, with the finite norms

$$
\begin{gathered}
\|f\|_{W L_{p, \lambda, \gamma}}=\sup _{r>0} r \sup _{t, x>0}\left(t^{-\gamma}|\{y \in H(x, t):|f(\operatorname{ch} y)|>r\}|_{\gamma}\right)^{\frac{1}{p}}, \\
\|f\|_{W \widetilde{L}_{p, \lambda, \gamma}}=\sup _{r>0} r \sup _{t, x>0}\left([t]_{1}^{-\gamma}|\{y \in H(x, t):|f(\operatorname{ch} y)|>r\}|_{\gamma}\right)^{\frac{1}{p}},
\end{gathered}
$$

respectively. 
Note that $W L_{p, \lambda}\left(\mathbb{R}_{+}, G\right)=W L_{p, \lambda, 0}\left(\mathbb{R}_{+}, G\right)=W \widetilde{L}_{p, \lambda, 0}\left(\mathbb{R}_{+}, G\right), L_{p, \lambda, \gamma}\left(\mathbb{R}_{+}, G\right) \subset$ $W L_{p, \lambda, \gamma}\left(\mathbb{R}_{+}, G\right)$ and $\|f\|_{W L_{p, \lambda, \gamma}} \leq\|f\|_{L_{p, \lambda, \gamma}}, \widetilde{L}_{p, \lambda, \gamma}\left(\mathbb{R}_{+}, G\right) \subset W L_{p, \lambda, \gamma}\left(\mathbb{R}_{+}, G\right)$ and $\|f\|_{W \widetilde{L}_{p, \lambda, \gamma}} \leq\|f\|_{\widetilde{L}_{p, \lambda, \gamma}}$.

The generalized shift operator associated with the operator $G_{\lambda}$ is of the form (see $[6,9])$

$$
A_{\text {cht }}^{\lambda} f(\operatorname{ch} x)=\frac{\Gamma\left(\lambda+\frac{1}{2}\right)}{\Gamma(\lambda) \Gamma\left(\frac{1}{2}\right)} \int_{0}^{\pi} f(\operatorname{ch} x \operatorname{ch} t-\operatorname{sh} x \operatorname{sht} \cos \varphi)(\sin \varphi)^{2 \lambda-1} d \varphi .
$$

This operator possesses properties similar to those of the generalized shift operator in Levitan's works [13] and [14].

By analogy with [16], we introduce the following notation.

Definition 2.1. Let $1 \leq p<\infty$ and let $w: \mathbb{R}_{+} \rightarrow \mathbb{R}_{+}$be a Lebesgue measurable function. The generalized Gegenbauer-Morrey ( $G$-Morrey) space $M_{p, \lambda, w}\left(\mathbb{R}_{+}, G\right)$ associated with the Gegenbauer differential operator $G_{\lambda}$ are the set of locally integrable functions $f(\operatorname{ch} x), x \in \mathbb{R}_{+}$with the finite norm

$$
\|f\|_{M_{p, \lambda, w}\left(\mathbb{R}_{+}, G\right)} \equiv\|f\|_{M_{p, \lambda, w}}:=\sup _{x \in \mathbb{R}_{+}, r>0}\left(\frac{1}{w(r)} \int_{H(0, r)} A_{c h t}^{\lambda}|f(c h x)|^{p} \operatorname{sh}^{2 \lambda} t d t\right)^{\frac{1}{p}},
$$

and the weak Morrey space $W M_{p, \lambda, w}\left(\mathbb{R}_{+}, G\right)$ are the set of locally integrable functions $f(\operatorname{ch} x), x \in \mathbb{R}_{+}$, with the finite norm

$$
\begin{aligned}
& \|f\|_{W M_{p, \lambda, w}}\left(\mathbb{R}_{+}, G\right) \equiv\|f\|_{W M_{p, \lambda, w}} \\
& =\sup _{r>0} r \sup _{x \in \mathbb{R}_{+}, t>0}\left(\frac{1}{w(t)}\left|\left\{y \in H(0, t):\left|A_{c h y}^{\lambda} f(\operatorname{ch} x)\right|>r\right\}\right|_{\lambda}\right)^{\frac{1}{p}} \\
& =\sup _{r>0} r \sup _{x \in \mathbb{R}_{+}, t>0}\left(\frac{1}{w(t)} \int_{\left\{y \in H(0, t): A_{c h y}^{\lambda}|f(\operatorname{ch} x)|>r\right\}} s h^{2 \lambda} y d y\right)^{\frac{1}{p}} .
\end{aligned}
$$

Under the choice $w(r)=r^{\gamma}, 0 \leq \gamma \leq 2 \lambda+1$, or $w(r)=[r]_{1}^{\gamma}$, we can write that $\left.L_{p, \lambda, \gamma}\left(\mathbb{R}_{+}, G\right) \equiv M_{p, \lambda, w}\left(\mathbb{R}_{+}, G\right)\right|_{w(r)=r^{\gamma}}$, and $\left.\widetilde{L}_{p, \lambda, \gamma}\left(\mathbb{R}_{+}, G\right) \equiv M_{p, \lambda, w}\left(\mathbb{R}_{+}, G\right)\right|_{w(r)=[r]_{1}^{\gamma}}$, respectively (see [5]).

Let $M_{G}$ be the Gegenbauer maximal operator(see [9]) for $f \in L_{1, \lambda}^{\text {loc }}\left(\mathbb{R}_{+}\right)$

$$
M_{G}(\operatorname{ch} x)=\sup _{r>0} \frac{1}{|H(0, r)|_{\lambda}} \int_{H(0, r)} A_{c h t}^{\lambda}|f(\operatorname{ch} x)| s h^{2 \lambda} t d t,
$$

where $|H(0, r)|_{\lambda}=\int_{0}^{r} s h^{2 \lambda} t d t$.

For $q \geq 1$ let

$$
M_{G}^{q} f(\operatorname{ch} x)=\left(M_{G}|f|^{q}(\operatorname{ch} x)\right)^{\frac{1}{q}} .
$$

The Riesz-Gegenbauer ((R-G)-potential) $I_{G}^{\alpha}$ is defined as follows (see $[3,4,5]$ )

$$
I_{G}^{\alpha} f(\operatorname{ch} x)=\frac{1}{\Gamma\left(\frac{\alpha}{2}\right)} \int_{0}^{\infty}\left(\int_{0}^{\infty} r^{\frac{\alpha}{2}-1} h_{r}(\operatorname{cht}) d r\right) A_{c h t}^{\lambda} f(\operatorname{ch} x) \operatorname{sh}^{2 \lambda} t d t,
$$

where

$$
h_{r}(\operatorname{ch} t)=\int_{1}^{\infty} e^{-u(u+2 \lambda) r} P_{u}^{\lambda}(\operatorname{ch} t) \operatorname{sh}^{2 \lambda} u d u
$$

and $P_{u}^{\lambda}(c h t)$ is an eigen function of the operator $G$. 
Throughout in the paper, we will denote by $\operatorname{sh} x, \operatorname{ch} x$ the hyperbolic functions and by $A \lesssim B$ we mean that $A \leq C B$ with some positive constant $C$ which can depend on some parameters. If $A \lesssim B$ and $B \lesssim A$, we write $A \approx B$ and say that they are equivalent.

\section{Main results}

Let $0<\delta \leq 1$. Assume that $w(r)$ satisfies the conditions: for any $r>0$

$$
\begin{gathered}
r \leq t \leq 2 r \Rightarrow w(t) \approx w(r), \\
\int_{r}^{\infty} \frac{w(t)}{t^{\gamma \delta+1}} d t \lesssim\left\{\begin{array}{l}
r^{-(2 \lambda+1) \delta} w(r), \gamma=2 \lambda+1 ; 0<r<2 . \\
r^{-4 \lambda \delta} w(r), \gamma=4 \lambda ; 2 \leq r<\infty .
\end{array}\right.
\end{gathered}
$$

Theorem 3.1. Let conditions (3.1) and (3.2) be valid. Then

(i) For $f \in M_{p, \lambda, w}\left(\mathbb{R}_{+}, G\right)$ and $1 \leq q<p<\infty$

$$
\left\|M_{G}^{q} f\right\|_{M_{p, \lambda, w}} \lesssim\|f\|_{M_{p, \lambda, w}} .
$$

(ii) For $f \in W M_{p, \lambda, w}\left(\mathbb{R}_{+}, G\right), 1 \leq p<\infty$ and for any $t>0$

$$
\left\|M_{G}^{p} f\right\|_{W M_{p, \lambda, w}} \lesssim\|f\|_{M_{p, \lambda, w}} .
$$

Now, we consider the Riesz-Gegenbauer potential ((R-G)-potential) $I_{G}^{\alpha}$.

Theorem 3.2. Let $0<\lambda<\frac{1}{2}, 0<\alpha<2 \lambda+1,1 \leq p<\frac{\alpha}{2 \lambda+1}$ and $\frac{1}{p}-\frac{1}{q}=\frac{\alpha}{2 \lambda+1}$. Assume that $w$ satisfies the conditions (3.1) and (3.2). Then

(i) if $p>1$ then for $f \in M_{p, \lambda, w}\left(\mathbb{R}_{+}, G\right)$

$$
\left\|I_{G}^{\alpha} f\right\|_{M_{q, \lambda, w} \frac{q}{p}} \lesssim\|f\|_{M_{p, \lambda, w}},
$$

(ii) if $p=1$ and $f \in M_{1, \lambda, w}\left(\mathbb{R}_{+}, G\right)$. Then

$$
\left\|I_{G}^{\alpha} f\right\|_{W M_{q, \lambda, w}} \lesssim\|f\|_{M_{1, \lambda, w}} .
$$

Corollary 3.1. [3] Let $0<\alpha<2 \lambda+1,0<\gamma<2 \lambda+1-\alpha$ and $1 \leq p<\frac{2 \lambda+1-\gamma}{\alpha}$.

(i) If $1<p<\frac{2 \lambda+1-\gamma}{\alpha}$, then condition $\frac{1}{p}-\frac{1}{q}=\frac{\alpha}{2 \lambda+1-\gamma}$ is necessary and sufficient for the boundedness of $I_{G}^{\alpha}$ from $L_{p, \lambda, \gamma}\left(\mathbb{R}_{+}, G\right)$ to $L_{q, \lambda, \gamma}\left(\mathbb{R}_{+}, G\right)$.

(ii) If $p=1<\frac{2 \lambda+1-\gamma}{\alpha}$, then the condition $1-\frac{1}{q}=\frac{\alpha}{2 \lambda+1-\gamma}$ is necessary and sufficient for the boundedness of $I_{G}^{\alpha}$ from $L_{1, \lambda, \gamma}\left(\mathbb{R}_{+}, G\right)$ to $W L_{q, \lambda, \gamma}\left(\mathbb{R}_{+}, G\right)$.

Corollary 3.2. [5] Let $0 \leq \alpha<2 \lambda+1,0 \leq \gamma<2 \lambda+1-\alpha$ and $1 \leq p<\frac{2 \lambda+1-\gamma}{\alpha}$.

1) If $1<p<\frac{2 \lambda+1-\gamma}{\alpha}$, then the condition $\frac{\alpha}{2 \lambda+1} \leq \frac{1}{p}-\frac{1}{q} \leq \frac{\alpha}{2 \lambda+1-\gamma}$ is necessary and sufficient for the boundedness of $I_{G}^{\alpha}$ from $\widetilde{L}_{p, \lambda, \gamma}\left(\mathbb{R}_{+}, G\right)$ to $\widetilde{L}_{q, \lambda, \gamma}\left(\mathbb{R}_{+}, G\right)$.

2) If $p=1<\frac{2 \lambda+1-\gamma}{\alpha}$, then the condition $\frac{\alpha}{2 \lambda+1} \leq 1-\frac{1}{q} \leq \frac{\alpha}{2 \lambda+1-\gamma}$ is necessary and sufficient for the boundedness of $I_{G}^{\alpha}$ from $\widetilde{L}_{1, \lambda, \gamma}\left(\mathbb{R}_{+}, G\right)$ to $W \widetilde{L}_{q, \lambda, \gamma}\left(\mathbb{R}_{+}, G\right)$. 


\section{Auxiliary results}

Further we need the following results.

Lemma 4.1. [9] For $0<\lambda<\frac{1}{2}$ the following relations are true:

$$
|H(0, r)|_{\lambda} \approx\left\{\begin{array}{l}
\left(\operatorname{sh} \frac{r}{2}\right)^{2 \lambda+1}, 0<r<2 \\
\left(\operatorname{ch} \frac{r}{2}\right)^{4 \lambda}, 2 \leq r<\infty
\end{array}\right.
$$

Let $\chi_{H}$ be the characteristic function of $H=H(0, r)$.

Lemma 4.2. [10]. For $x \in \mathbb{R}_{+}, r>0$, and $0<\lambda<\frac{1}{2}$ the following relation

$$
M_{G} \chi_{H}(\operatorname{ch} x) \approx\left\{\begin{array}{l}
\left(\frac{\operatorname{sh} \frac{r}{2}}{\operatorname{sh} \frac{x+r}{2}}\right)^{2 \lambda+1}, 0<x+r<2, \\
\left(\frac{\operatorname{sh} \frac{r}{2}}{\operatorname{sh} \frac{x+r}{2}}\right)^{4 \lambda}, 2 \leq x+r<\infty
\end{array}\right.
$$

is valid.

Lemma 4.3. For every nonnegative function $f(\operatorname{ch} x), x \in \mathbb{R}_{+}$the following relation

$$
\int_{0}^{r} A_{c h t}^{\lambda} f(\operatorname{ch} x) s h^{2 \lambda} t d t \approx \int_{H(x, r)} f(\operatorname{ch} u) s h^{2 \lambda} u d u
$$

is valid.

Proof. In the work [9] it is proved that (see [9], proof of Theorem 2.1)

$$
\begin{gathered}
J(x, r)=\int_{0}^{r} A_{c h t}^{\lambda} f(c h x) s h^{2 \lambda} t d t \\
=C_{\lambda} \int_{c h(x-r)}^{\operatorname{ch}(x+r)} f(z)\left(z^{2}-1\right)^{\lambda-\frac{1}{2}} \int_{\varphi(z, x, r)}\left(1-u^{2}\right)^{\lambda-1} d u d z,
\end{gathered}
$$

where $\varphi(z, x, r)=\frac{z \operatorname{ch} x-\operatorname{ch} r}{\sqrt{z^{2}-1} \operatorname{sh} x}$ and $-1 \leq \varphi(z, x, r) \leq 1, C_{\lambda}=\frac{\Gamma\left(\lambda+\frac{1}{2}\right)}{\Gamma(\lambda) \Gamma\left(\frac{1}{2}\right)}$.

Then

$$
A(z, x, r)=C_{\lambda} \int_{\varphi(z, x, r)}^{1}\left(1-u^{2}\right)^{\lambda-1} d u \leq C_{\lambda} \int_{-1}^{1}\left(1-u^{2}\right)^{\lambda-1} d u=1 .
$$

Now estimate the integral $A(z, x, r)$. Let $-1 \leq \varphi(z, x, r) \leq 0$. Then

$$
\begin{aligned}
A(z, x, r) & =C_{\lambda} \int_{\varphi(z, x, r)}^{1}\left(1-u^{2}\right)^{\lambda-1} d u \geq C_{\lambda} \int_{0}^{1}\left(1-u^{2}\right)^{\lambda-1} d u \\
& \geq 2^{\lambda-1} C_{\lambda} \int_{0}^{1}(1-u)^{\lambda-1} d u=\frac{2^{\lambda-1}}{\lambda} C_{\lambda} .
\end{aligned}
$$


Now, let $0 \leq \varphi(z, x, r) \leq 1$, then

$$
\begin{aligned}
& A(z, x, r)=C_{\lambda} \int_{\varphi(z, x, r)}^{1}(1-u)^{\lambda-1}(1+u)^{\lambda-1} d u=C_{\lambda} \int_{0}^{1-\varphi(z, x, r)} u^{\lambda-1}(2-u)^{\lambda-1} d u \\
& =C_{\lambda} \int_{\frac{1}{1-\varphi(z, x, r)}}^{\infty} u^{-\lambda-1}\left(2-\frac{1}{u}\right)^{\lambda-1} d u=C_{\lambda} \int_{\frac{1}{1-\varphi(z, x, r)}}^{\infty} u^{-2 \lambda}(2 u-1)^{\lambda-1} d u \\
& =2^{2 \lambda-1} C_{\lambda} \int_{\frac{2}{1-\varphi(z, x, r)}}^{\infty} u^{-2 \lambda}(u-1)^{\lambda-1} d u=2^{2 \lambda-1} C_{\lambda} \int_{\frac{1-\varphi(z, x, r)}{1+\varphi(z, x) r}}^{\infty}(u+1)^{-2 \lambda} u^{\lambda-1} d u \\
& =2^{2 \lambda-1} \cdot C_{\lambda} \int_{0}^{\frac{1+\varphi(z, x, r)}{1-\varphi(z, x, r)}}(1+u)^{-2 \lambda} u^{\lambda-1} d u \geq 2^{2 \lambda-1} C_{\lambda} \int_{0}^{1}(1+u)^{-2 \lambda} u^{\lambda-1} d u \\
& \geq 2^{2 \lambda-1} C_{\lambda} \int_{0}^{1} \frac{u^{\lambda-1}}{(1+u)^{2 \lambda}} d u \geq \frac{C_{\lambda}}{2} \int_{0}^{1} u^{\lambda-1} d u=\frac{C_{\lambda}}{2 \lambda} .
\end{aligned}
$$

Consequently,

$$
A(z, x, r)=\int_{\varphi(z, x, r)}^{1}\left(1-u^{2}\right)^{\lambda-1} d u \approx 1
$$

and

$$
J(x, r) \approx \int_{c h(x-r)}^{\operatorname{ch}(x+r)} f(z)\left(z^{2}-1\right)^{\lambda-\frac{1}{2}} d z=\int_{H(x, r)} f(\operatorname{chu}) \operatorname{sh}^{2 \lambda} u d u .
$$

Theorem 4.1. (Calderon-Zygmund decomposition of $\mathbb{R}^{n}$ ). Suppose that $f$ is nonnegative integrable on $\mathbb{R}^{+}$. Then for any fixed $\alpha>0$, there exists a sequence $\left\{H_{j}\left(x_{j}, r_{j}\right)\right\}=\left\{H_{j}\right\}$ of disjoint interval such that

(1) $f(\operatorname{ch} x) \leq \alpha$ for a.e. $x \notin \bigcup_{j} H_{j}$;

(2) $\left|\bigcup_{j} H_{j}\right|_{\lambda} \leq \frac{1}{\alpha}\|f\|_{L_{1, \lambda}}$;

(3) $\alpha<\frac{1}{\left|H_{j}\right|_{\lambda}} \int_{H_{j}} f($ chy $) s h^{2 \lambda} y d y \lesssim 2^{(2 \lambda+1) n} \alpha, n=1,2, \ldots$

The proof of this theorem is similar to Theorem 1.2.1 from [15].

Theorem 4.2. (Fefferman-Stein type inequality) 
(i) For every nonnegative measurable functions $f$ and $g$ on $\mathbb{R}_{+}$every $1 \leq p<\infty$ and every $0<t<\infty$,

$$
\int_{\mathbb{R}_{+}} A_{c h t}^{\lambda}\left(M_{G} f(\operatorname{ch} x)\right)^{p} g(\operatorname{ch} x) \operatorname{sh}^{2 \lambda} x d x \lesssim \int_{\mathbb{R}_{+}} A_{c h t}^{\lambda} f(\operatorname{ch} x)^{p} M_{G} g(\operatorname{ch} x) \operatorname{sh}^{2 \lambda} x d x,
$$

(ii) For any measurable function on $\mathbb{R}_{+} f \geq 0$ and $g \geq 0$

$$
\int_{\left\{x \in \mathbb{R}_{+}: A_{c h t}^{\lambda} M_{G} f(\operatorname{ch} x)>\alpha\right\}} g(\operatorname{ch} x) \operatorname{sh}^{2 \lambda} x d x \lesssim \frac{1}{\alpha} \int_{\mathbb{R}_{+}} A_{c h t}^{\lambda} f(\operatorname{ch} x) M_{G} g(\operatorname{ch} x) \operatorname{sh}^{2 \lambda} x d x,
$$

Proof. First assertion follows from the inequality (see[3], Theorem 1.4)

$$
\int_{0}^{r} A_{\text {cht }}^{\lambda}\left(M_{G} f(\operatorname{ch} x)\right)^{p} g(\operatorname{ch} x) \operatorname{sh}^{2 \lambda} x d x \lesssim \int_{0}^{r} A_{\text {cht }}^{\lambda} f(\operatorname{ch} x)^{p} M_{G} g(\operatorname{ch} x) \operatorname{sh}^{2 \lambda} x d x
$$

as $r \rightarrow \infty$.

We prove (4.2). Using the relation from Lemma 4.3

$$
\int_{H(0, r)} A_{c h t}^{\lambda} f(\operatorname{ch} x) s h^{2 \lambda} t d t \approx \int_{H(x, r)} f(c h u) s h^{2 \lambda} u d u \approx \alpha
$$

we obtain

$$
\begin{aligned}
& \int_{H_{i}(0, r)} A_{c h t}^{\lambda} f(\operatorname{ch} x) M_{G} g(\operatorname{ch} x) \operatorname{sh}^{2 \lambda} x d x \\
& \geq \int_{H_{i}(0, r)} A_{c h t}^{\lambda} f(\operatorname{ch} x)\left(\frac{1}{\left|H_{i}(0, r)\right|_{\lambda}} \int_{H_{i}(0, r)} A_{c h t}^{\lambda} g(\operatorname{ch} x) s h^{2 \lambda} y d y\right) s h^{2 \lambda} x d x \\
& \geq \alpha \int_{\left\{u \in R_{+}: M_{G} f(\operatorname{chu})>\alpha\right\}} g(\operatorname{chu}) \operatorname{sh}^{2 \lambda} u d u .
\end{aligned}
$$

Summing over $i$, we get

$$
\begin{aligned}
\int_{\mathbb{R}_{+}} A_{c h t}^{\lambda} f(\operatorname{ch} x) M_{G} g(\operatorname{ch} x) \operatorname{sh}^{2 \lambda} x d x & \gtrsim \alpha \int_{\mathbb{R}_{+}} g(\operatorname{chu}) s h^{2 \lambda} u d u \\
& \gtrsim \alpha \int_{\left\{u \in \mathbb{R}_{+}: M_{G} f(\operatorname{chu})>\alpha\right\}} g(\operatorname{chu}) \operatorname{sh}^{2 \lambda} u d u .
\end{aligned}
$$

From this it follows (4.2).

Lemma 4.4. Let the conditions (3.1) and (3.2) hold.

Then for $1 \leq p<\infty$ and $f \in M_{p, \lambda, w}\left(\mathbb{R}_{+}, G\right)$ we have

$$
\int_{\mathbb{R}_{+}} A_{c h t}^{\lambda}|f(\operatorname{ch} x)|^{p}\left(M_{G} \chi_{H}(\operatorname{ch} x)\right)^{\delta} s h^{2 \lambda} x d x \lesssim w(r)\|f\|_{M_{p, \lambda, w}}^{p} .
$$


Proof. Let $\chi_{H}$ be the characteristic function of $H(0, r)$. Then $M_{G} \chi_{H} \leq 1$. On the other hand, by Lemma 4.2 for $0<x+r<2$ we have

$$
\begin{aligned}
& \int_{\mathbb{R}_{+}} A_{c h t}^{\lambda}|f(\operatorname{ch} x)|^{p}\left(M_{G} \chi_{H}(\operatorname{cht})\right)^{\delta} s h^{2 \lambda} t d t \\
& \approx \int_{0}^{r} A_{c h t}^{\lambda}|f(\operatorname{ch} x)|^{p} s h^{2 \lambda} t d t \\
& +\sum_{k=0}^{\infty} \int_{2^{k} r}^{2^{k+1} r}\left(\frac{s h \frac{r}{2}}{s h \frac{x+r}{2}}\right)^{(2 \lambda+1) \delta} A_{c h t}^{\lambda}|f(\operatorname{ch} x)|^{p} s h^{2 \lambda} t d t \\
& \approx w(r)\left(\frac{1}{w(r)} \int_{0}^{r} A_{c h t}^{\lambda}|f(c h x)|^{p} s h^{2 \lambda} t d t\right) \\
& +\sum_{k=0}^{\infty} \int_{2^{k} r}^{2^{k+1} r}\left(\frac{s h \frac{r}{2}}{s h\left(2^{k+1}+1\right) \frac{r}{2}}\right)^{(2 \lambda+1) \delta} A_{c h t}^{\lambda}|f(\operatorname{ch} x)|^{p} s h^{2 \lambda} t d t
\end{aligned}
$$

(since $\operatorname{sh} a x \geq a \operatorname{sh} x$ for $a \geq 1$ )

$$
\begin{aligned}
& \lesssim\left(w(r)+\sum_{k=0}^{\infty} 2^{-(2 \lambda+1) \delta} w\left(2^{k+1} r\right)\right)\|f\|_{M_{p, \lambda, w}}^{p} \\
& \lesssim\left(r^{(2 \lambda+1) \delta} \sum_{k=0}^{\infty} \frac{w\left(2^{k} r\right)}{(2 \lambda+1)^{(2 \lambda+1) \delta}}\right)\|f\|_{M_{p, \lambda, w}}^{p}
\end{aligned}
$$

By (3.1)

$$
\frac{w\left(2^{k} r\right)}{\left(2^{k} r\right)^{(2 \lambda+1) \delta}} \lesssim \int_{2^{k} r}^{2^{k+1} r} \frac{w(t)}{t^{(2 \lambda+1) \delta+1}} d t
$$

we have

$$
\begin{aligned}
& \int_{\mathbb{R}_{+}} A_{c h t}^{\lambda}|f(\operatorname{ch} x)|^{p}\left(M_{G} \chi_{H}(\operatorname{cht})\right)^{\delta} s h^{2 \lambda} t d t \\
& \lesssim\left(r^{(2 \lambda+1) \delta} \int_{r}^{\infty} \frac{w(t)}{t^{(2 \lambda+1) \delta+1}} d t\right)\|f\|_{M_{p, \lambda, w}^{p}} \lesssim w(r)\|f\|_{M_{p, \lambda, w}^{p}} .
\end{aligned}
$$

If $2 \leq x+r<\infty$, then by Lemma 4.2 and previous case we obtain

$$
\begin{aligned}
& \int_{\mathbb{R}_{+}} A_{c h t}^{\lambda}|f(\operatorname{ch} x)|^{p}\left(M_{G} \chi_{H}(c h t)\right)^{\delta} s h^{2 \lambda} t d t \\
& \lesssim\left(r^{4 \lambda} \int_{r}^{\infty} \frac{w(t)}{t^{4 \lambda \delta+1}} d t\right)\|f\|_{M_{p, \lambda, w}} \lesssim w(r)\|f\|_{M_{p, \lambda, w}} .
\end{aligned}
$$

Now the assertion of Lemma 3.4 follows from (4.3) and (4.4).

\section{Proofs of the main results}

Proof of Theorem 3.1. (i) We use (4.1) for $|f|^{q}$ and $\chi_{H} \geq 0$, the characteristic function of $H(0, r)$.

Then

$$
\int_{H(0, r)} A_{c h t}^{\lambda}\left(M_{G}^{q} f(\operatorname{ch} x)\right)^{p} \operatorname{sh}^{2 \lambda} x d x \lesssim \int_{\mathbb{R}_{+}} A_{c h t}^{\lambda}|f(\operatorname{ch} x)|^{p} M_{G} \chi_{H}(\operatorname{ch} x) \operatorname{sh}^{2 \lambda} x d x
$$


It follows from Lemma 4.4 with $\delta=1$ that

$$
\int_{H(0, r)} A_{c h t}^{\lambda}\left(M_{G}^{q} f(\operatorname{ch} x)\right)^{p} s h^{2 \lambda} x d x \lesssim w(r)\|f\|_{M_{p, \lambda, w}} .
$$

Therefore we obtain (3.3).

(ii) We use (4.2). By Lemma 4.4 with $\delta=1$ we have

$$
\begin{aligned}
& \left|\left\{x \in H(0, r): A_{c h t}^{\lambda} M_{G} f(\operatorname{ch} x)>\alpha\right\}\right|_{\lambda} \\
& =\int_{\left\{x \in \mathbb{R}_{+}: A_{c h t}^{\lambda} M_{G}|f|^{p}(\operatorname{ch} x)>\alpha^{p}\right\}} \chi_{H}(\operatorname{ch} x) s h^{2 \lambda} x d x \\
& \lesssim \alpha^{-p} \int_{\mathbb{R}_{+}} A_{c h t}^{\lambda}|f(\operatorname{ch} x)|^{p} M \chi_{H}(\operatorname{ch} x) s h^{2 \lambda} x d x \\
& \lesssim \alpha^{-p} w(r)\|f\|_{M_{p, \lambda, w}}^{p} .
\end{aligned}
$$

From this it follows (3.4).

To prove Theorem 3.2 we need the following result (see [4], Theorem 3)

Theorem 5.1. [4] Let $0<\lambda<\frac{1}{2}, 0<\alpha<2 \lambda+1$ and $1 \leq p<\frac{2 \lambda+1}{\alpha}$.

(a) If $1<p<\frac{2 \lambda+1}{\alpha}$, then the condition $\frac{1}{p}-\frac{1}{q}=\frac{\alpha}{2 \lambda+1}$ is necessary and sufficient for the boundedness of the operator $I_{G}^{\alpha}$ from $L_{p, \lambda}\left(\mathbb{R}_{+}, G\right)$ to $L_{q, \lambda}\left(\mathbb{R}_{+}, G\right)$.

(b) If $p=1$, the condition is necessary and sufficient for the boundedness of the operator $I_{G}^{\alpha}$ from $L_{1, \lambda}\left(\mathbb{R}_{+}, G\right)$ to $L_{q, \lambda}\left(\mathbb{R}_{+}, G\right)$.

Proof of Theorem 3.2. (i) For $f \in M_{p, \lambda, w}\left(\mathbb{R}_{+}, G\right)$ and for $H(0, r)$, let $f=f_{1}+f_{2}, f_{1}=f \chi_{H}$. Since $I_{G}^{\alpha}$ is bounded from $L_{p, \lambda}\left(\mathbb{R}_{+}, G\right)$ to $L_{q, \lambda}\left(\mathbb{R}_{+}, G\right)$,

$$
\begin{aligned}
& \int_{H(0, r)} A_{c h t}^{\lambda}\left|I_{G}^{\alpha} f_{1}(\operatorname{ch} x)\right|^{q} s h^{2 \lambda} x d x \lesssim\left\|I_{G}^{\alpha} f_{1}\right\|_{L_{q, \lambda}(H(0, r))}^{q} \\
& \lesssim\left\|f_{1}\right\|_{L_{q, \lambda}(H(0, r))}^{q} \lesssim\left(\int_{H(0, r)} A_{c h t}^{\lambda}|f(\operatorname{ch} x)|^{p} \operatorname{sh}^{2 \lambda} x d x\right)^{\frac{q}{p}} .
\end{aligned}
$$

Therefore,

$$
\begin{gathered}
\left(w(r)^{-\frac{q}{p}} \int_{H(0, r)} A_{c h t}^{\lambda}\left|I_{G}^{\alpha} f_{1}(\operatorname{ch} x)\right|^{q} s h^{2 \lambda} x d x\right)^{\frac{1}{q}} \\
\lesssim\left(\frac{1}{w(r)} \int_{H(0, r)} A_{c h t}^{\lambda}|f(\operatorname{ch} x)|^{p} s h^{2 \lambda} t d t\right)^{\frac{1}{p}} \lesssim\|f\|_{M_{p, \lambda, w}} .
\end{gathered}
$$

For $x \in H(0, r)$ and for $t \in(r, \infty)$ we have

$$
\begin{gathered}
\left|I_{G}^{\alpha} f_{2}(\operatorname{ch} x)\right| \lesssim\left\{\begin{array}{l}
\int_{r}^{\infty} \frac{A_{c h t}^{\lambda}\left|f_{2}(c h x)\right| s h^{2 \lambda} t}{(c h t)^{2 \lambda+1}} d t, 0<r<2, \\
\int_{r}^{\infty} \frac{A_{c h t}^{\lambda}\left|f_{2}(c h x)\right| s h^{2 \lambda} t}{(c h t)^{4 \lambda}} d t, 2<r<\infty,
\end{array}\right. \\
\lesssim\left\{\begin{array}{l}
\int_{r}^{\infty} \frac{A_{c h t}^{\lambda} \mid f_{2}\left(\operatorname{chx} x \mid s h^{2 \lambda} t\right.}{(c h t)^{2 \lambda+1-\alpha}} d t, 0<r<2, \\
\int_{r}^{\infty} \frac{A_{c h t}^{\lambda}\left|f_{2}(\operatorname{chx})\right| s h^{2 \lambda} t}{(c h t)^{4 \lambda-\alpha}} d t, 2<r<\infty,
\end{array}\right.
\end{gathered}
$$




$$
\begin{gathered}
\lesssim\left\{\begin{array}{l}
\left(s h \frac{r}{2}\right)^{\alpha-2 \lambda+1} \int_{r}^{\infty} A_{c h t}^{\lambda}\left|f_{2}(\operatorname{ch} x)\right|\left(\frac{s h \frac{r}{2}}{s h \frac{t+r}{2}}\right)^{2 \lambda+1-\alpha} s h^{2 \lambda} t d t, 0<r<2, \\
\left(s h \frac{r}{2}\right)^{\alpha-4 \lambda} \int_{r}^{\infty} A_{c h t}^{\lambda}\left|f_{2}(\operatorname{ch} x)\right|\left(\frac{s h \frac{r}{2}}{s h \frac{t+r}{2}}\right)^{4 \lambda-\alpha} s h^{2 \lambda} t d t, 2<r<\infty,
\end{array}\right. \\
\lesssim\left\{\begin{array}{l}
\left(s h \frac{r}{2}\right)^{-(2 \lambda+1)\left(1-\frac{\alpha}{2 \lambda+1}\right)} \int_{r}^{\infty} A_{c h t}^{\lambda}\left|f_{2}(\operatorname{ch} x)\right|\left(\frac{s h \frac{r}{2}}{s h \frac{t+r}{2}}\right)^{(2 \lambda+1)\left(1-\frac{\alpha}{2 \lambda+1}\right)} s h^{2 \lambda} t d t, 0<r<2, \\
\left(s h \frac{r}{2}\right)^{-4 \lambda\left(1-\frac{\alpha}{4 \lambda}\right)} \int_{r}^{\infty} A_{c h t}^{\lambda}\left|f_{2}(\operatorname{ch} x)\right|\left(\frac{s h \frac{r}{2}}{s h \frac{t+r}{2}}\right)^{4 \lambda\left(1-\frac{\alpha}{4 \lambda}\right)} s h^{2 \lambda} t d t, 2<r<\infty,
\end{array}\right. \\
\approx\left\{\begin{array}{l}
|H(0, r)|_{\lambda}^{\frac{\alpha}{2 \lambda+1}}-1 \int_{r}^{\infty} A_{c h t}^{\lambda}\left|f_{2}(\operatorname{ch} x)\right|\left(M_{G} \chi_{H}(\operatorname{cht} t)\right)^{1-\frac{\alpha}{2 \lambda+1}} s h^{2 \lambda} t d t, 0<r<2 \\
|H(0, r)|_{\lambda}^{\frac{\alpha}{4 \lambda}-1} \int_{r}^{\infty} A_{c h t}^{\lambda}\left|f_{2}(\operatorname{ch} x)\right|\left(M_{G} \chi_{H}(\operatorname{cht} t)^{1-\frac{\alpha}{4 \lambda}} s h^{2 \lambda} t d t, 2<r<\infty .\right.
\end{array}\right.
\end{gathered}
$$

First we consider the case $0<r<2$ and $0<\alpha<2 \lambda+1$. Let $0<\delta<1-\frac{\alpha p}{2 \lambda+1}$. By Hölder's inequality, we have

$$
\begin{aligned}
& \left|I_{G}^{\alpha} f_{2}(\operatorname{ch} x)\right| \\
& \lesssim \frac{1}{|H(0, r)|_{\lambda}^{1-\frac{\alpha}{2 \lambda+1}}} \int_{r}^{\infty} A_{c h t}^{\lambda}\left|f_{2}(\operatorname{ch} x)\right|\left(M_{G} \chi_{H}(\operatorname{ch} x)\right)^{\frac{\delta}{p}}\left(M_{G} \chi_{H}(c h t)\right)^{1-\frac{\alpha}{2 \lambda+1}-\frac{\delta}{p}} s h^{2 \lambda} t d t \\
& \lesssim \frac{1}{|H(0, r)|_{\lambda}^{1-\frac{\alpha}{2 \lambda+1}}}\left(\int_{r}^{\infty} A_{c h t}^{\lambda}\left|f_{2}(\operatorname{chx})\right|^{p}\left(M_{G} \chi_{H}(c h t)\right)^{\delta} s h^{2 \lambda} t d t\right)^{\frac{1}{p}} \\
& \times\left(\int_{r}^{\infty}\left(M_{G} \chi_{H}(c h t)\right)^{p-\frac{\alpha p}{2 \lambda+1}-\delta} s h^{2 \lambda} t d t\right)^{\frac{p-1}{p}} \\
& =\frac{1}{|H(0, r)|_{\lambda}^{1-\frac{1}{p}+\frac{1}{q}}}\left(\int_{r}^{\infty} A_{c h t}^{\lambda}\left|f_{2}(\operatorname{chx})\right|^{p}\left(M_{G} \chi_{H}(\operatorname{cht})\right)^{\delta} s h^{2 \lambda} t d t\right)^{\frac{1}{p}} \\
& \times\left(\int_{r}^{\infty}\left(M_{G} \chi_{H}(c h t)\right)^{\frac{p-\frac{\alpha p}{2 \lambda+1}-\delta}{p-1}} s h^{2 \lambda} t d t\right)^{\frac{p-1}{p}} \\
& =\frac{1}{|H(0, r)|_{\lambda}^{\frac{1}{q}}}\left(\int_{r}^{\infty} A_{c h t}^{\lambda} \mid f_{2}\left(\left.\operatorname{chs} x\right|^{p}\left(M_{G} \chi_{H} \operatorname{cht}\right)^{\delta} s h^{2 \lambda} t d t\right)^{\frac{1}{p}}\right. \\
& \times\left(\frac{1}{|H(0, r)|_{\lambda}} \int_{r}^{\infty}\left(M_{G} \chi_{H}(\operatorname{cht} t)\right)^{\frac{p-\frac{\alpha p}{2 \lambda+1}-\delta}{p-1}} s h^{2 \lambda} t d t\right)^{\frac{p-1}{p}}
\end{aligned}
$$

Further

$$
\begin{aligned}
& \frac{1}{|H(0, r)|_{\lambda}} \int_{r}^{\infty}\left(M_{G} \chi_{H}(c h t)\right)^{\frac{p-\frac{\alpha p}{2 \lambda+1}-\delta}{p-1}} s h^{2 \lambda} t d t \\
\approx & \frac{1}{|H(0, r)|_{\lambda}} \int_{r}^{\infty}\left(\frac{s h \frac{r}{2}}{s h \frac{t+r}{2}}\right)^{\frac{(2 \lambda+1)(p-\delta)-\alpha p}{p-1}} s h^{2 \lambda} t d t
\end{aligned}
$$




$$
\begin{gathered}
\lesssim \frac{\left(\operatorname{sh} \frac{r}{2}\right)^{\frac{(2 \lambda+1)(p-\delta)-\alpha p}{p-1}}}{\left(\operatorname{sh} \frac{r}{2}\right)^{2 \lambda+1}} \int_{r}^{\infty} \frac{\operatorname{sh}^{2 \lambda} \frac{t}{2} \operatorname{ch}^{2 \lambda} \frac{t}{2}}{\left(\operatorname{sh} \frac{t}{2}\right)^{\frac{(2 \lambda+1)(p-\delta)-\alpha p}{p-1}} d t} \\
\lesssim\left(\operatorname{sh} \frac{r}{2}\right)^{\frac{(2 \lambda+1)(p-\delta)-\alpha p}{p-1}-(2 \lambda+1)} \int_{r}^{\infty} \frac{\operatorname{sh}^{2 \lambda} \frac{t}{2} \operatorname{ch} \frac{t}{2}}{\left(\operatorname{sh} \frac{t}{2}\right)^{\frac{(2 \lambda+1)(p-\delta)-\alpha p}{p-1}}} d t \\
\lesssim\left(\operatorname{sh} \frac{r}{2}\right)^{\frac{(2 \lambda+1)(1-\delta)-\alpha p}{p-1}} \int_{r}^{\infty} \frac{d\left(\operatorname{sh} \frac{t}{2}\right)}{\left(\operatorname{sh} \frac{t}{2}\right)^{\frac{(2 \lambda+1)(p-\delta)-\alpha p}{p-1}-(2 \lambda+1)+1}} \\
\lesssim\left(\operatorname{sh} \frac{r}{2}\right)^{\frac{(2 \lambda+1)(1-\delta)-\alpha p}{p-1}} \int_{r}^{\infty} \frac{d\left(\operatorname{sh} \frac{t}{2}\right)}{\left(\operatorname{sh} \frac{t}{2}\right)^{\frac{(2 \lambda+1)(1-\delta)-\alpha p}{p-1}+1}} \lesssim 1 .
\end{gathered}
$$

From (5.3) and (5.4) we obtain

$$
\left|I_{G}^{\alpha} f_{2}(\operatorname{ch} x)\right| \lesssim|H(0, r)|_{\lambda}^{-\frac{1}{q}}\left\{\int_{r}^{\infty} A_{c h t}^{\lambda}\left|f_{2}(\operatorname{ch} x)\right|^{p}\left(M_{G} \chi_{H}(\operatorname{ch} x)\right)^{\delta} s h^{2 \lambda} t d t\right\}^{\frac{1}{p}}
$$

for $0<x+r<2$ and $0<\alpha<2 \lambda+1$.

Now we consider the case $2 \leq x+r<\infty$ and $0<\alpha \leq 4 \lambda$.

Let $0<\delta<1-\frac{\alpha p}{4 \lambda}$. By Hölder's inequality we have

\section{$\left|I_{G}^{\alpha} f_{2}(\operatorname{ch} x)\right|$}

$$
\begin{aligned}
& \lesssim \frac{1}{|H(0, r)|_{\lambda}^{1-\frac{\alpha}{4 \lambda}}} \int_{r}^{\infty} A_{c h t}^{\lambda}\left|f_{2}(\operatorname{ch} x)\right|\left(M_{G} \chi_{H}(\operatorname{cht} t)\right)^{\frac{\delta}{p}}\left(M_{G} \chi_{H}(\operatorname{cht} t)\right)^{1-\frac{\alpha}{4 \lambda}-\frac{\delta}{p}} s h^{2 \lambda} t d t \\
& \lesssim \frac{1}{|H(0, r)|_{\lambda}^{1-\frac{\alpha}{4 \lambda}}}\left(\int_{r}^{\infty} A_{c h t}^{\lambda}\left|f_{2}(\operatorname{ch} x)\right|^{p}\left(M_{G} \chi_{H}(\operatorname{cht} t)\right)^{\delta} s h^{2 \lambda} t d t\right)^{\frac{1}{p}} \\
& \times \frac{\left(\operatorname{sh} \frac{r}{2}\right)^{1-2 \lambda}}{\left(\operatorname{sh} \frac{r}{2}\right)^{2 \lambda+1-\alpha}}\left(\int_{r}^{\infty} A_{c h t}^{\lambda}\left|f_{2}(\operatorname{ch} x)\right|^{p}\left(M_{G} \chi_{H}(\operatorname{cht} t)\right)^{\frac{p-\frac{\alpha p}{4 \lambda}-\delta}{p-1}} s h^{2 \lambda} t d t\right)^{\frac{p-1}{p}} \\
& \lesssim \frac{\left(\operatorname{sh} \frac{r}{2}\right)^{1-2 \lambda}}{\left(\operatorname{sh} \frac{r}{2}\right)^{2 \lambda+1-\alpha}}\left(\int_{r}^{\infty} A_{c h t}^{\lambda}\left|f_{2}(\operatorname{ch} x)\right|^{p}\left(M_{G} \chi_{H}(\operatorname{cht} t)\right)^{\delta} s h^{2 \lambda} t d t\right)^{\frac{1}{p}} \\
& \times\left(\int_{r}^{\infty}\left(\frac{s h \frac{r}{2}}{s h \frac{t+r}{2}}\right)^{\frac{4 \lambda(p-\delta)-\alpha p}{p-1}} s h^{2 \lambda} t d t\right)^{\frac{p-1}{p}} \\
& \lesssim \frac{|H(0, r)|_{\lambda}^{\frac{1-2 \lambda}{1+2 \lambda}}}{|H(0, r)|_{\lambda}^{\frac{1}{q}+1-\frac{1}{q}}}\left(\int_{r}^{\infty} A_{c h t}^{\lambda}\left|f_{2}(\operatorname{ch} x)\right|^{p}\left(M_{G} \chi_{H}(c h t)\right)^{\delta} s h^{2 \lambda} t d t\right)^{\frac{1}{p}} \\
& \times \frac{\left(\operatorname{sh} \frac{r}{2}\right)^{1-2 \lambda}}{|H(0, r)|_{\lambda}^{\frac{1}{q}}}\left(\frac{1}{\left(\operatorname{sh} \frac{r}{2}\right)^{2 \lambda+1}} \int_{r}^{\infty} \frac{\operatorname{sh}^{2 \lambda} \frac{t}{2} \operatorname{ch}^{2 \lambda} \frac{t}{2}}{\left(\operatorname{sh} \frac{r}{2}\right)^{\frac{4 \lambda(p-\delta)-\alpha p}{p-1}}} d t\right)^{\frac{p-1}{p}}
\end{aligned}
$$




$$
\begin{aligned}
& \lesssim \frac{1}{|H(0, r)|_{\lambda}^{\frac{1}{q}}}\left(\int_{r}^{\infty} A_{c h t}^{\lambda}\left|f_{2}(\operatorname{ch} x)\right|^{p}\left(M_{G} \chi_{H}(\operatorname{cht} t)\right)^{\delta} s h^{2 \lambda} t d t\right)^{\frac{1}{p}} \\
& \quad \times\left(\operatorname{sh} \frac{r}{2}\right)^{1-2 \lambda}\left(\frac{1}{\left(\operatorname{sh} \frac{r}{2}\right)^{2 \lambda+1}} \int_{r}^{\infty} \frac{d\left(\operatorname{sh} \frac{t}{2}\right)}{\left(\operatorname{sh} \frac{t}{2}\right)^{\frac{4 \lambda(1-\delta)-\alpha p}{p-1}+1}}\right)^{\frac{p-1}{p}}
\end{aligned}
$$

We estimate the expression

$$
\frac{1}{\left(\operatorname{sh} \frac{r}{2}\right)^{2 \lambda+1}} \int_{r}^{\infty} \frac{d\left(\operatorname{sh} \frac{t}{2}\right)}{\left(\operatorname{sh} \frac{t}{2}\right)^{\frac{4 \lambda(1-\delta)-\alpha p}{p-1}+1}} \approx \frac{1}{\left(\operatorname{sh} \frac{r}{2}\right)^{2 \lambda+1+\frac{4 \lambda(1-\delta)-\alpha p}{p-1}}} .
$$

From (5.6) we get

$$
\begin{aligned}
& \left(\operatorname{sh} \frac{r}{2}\right)^{1-2 \lambda}\left(\frac{1}{\left(\operatorname{sh} \frac{r}{2}\right)^{2 \lambda+1}} \int_{r}^{\infty} \frac{d\left(\operatorname{sh} \frac{t}{2}\right)}{\left(\operatorname{sh} \frac{t}{2}\right)^{\frac{4 \lambda(1-\delta)-\alpha p}{p-1}+1}}\right)^{\frac{p-1}{p}} \\
& \lesssim \frac{1}{\left(\operatorname{sh} \frac{r}{2}\right)^{\frac{1}{p}(4 \lambda(1-\delta)+(4 \lambda-\alpha) p-2 \lambda-1)} \lesssim 1 .}
\end{aligned}
$$

From this and (5.6) we obtain

$$
\left|I_{G}^{\alpha} f_{2}(\operatorname{ch} x)\right| \lesssim \frac{1}{|H(0, r)|_{\lambda}^{\frac{1}{q}}}\left(\int_{r}^{\infty} A_{c h t}^{\lambda}\left|f_{2}(\operatorname{ch} x)\right|^{p}\left(M_{G} X_{H}(\operatorname{cht} t)\right)^{\delta} s h^{2 \lambda} t d t\right)^{\frac{1}{p}}
$$

for $2 \leq x+r<\infty$ and $0<\alpha \leq 4 \lambda$.

It remains to consider the case $2 \leq x+r<\infty$ and $4 \lambda<\alpha<2 \lambda+1$.

Let $\delta<1-\frac{(8 \lambda-\alpha) p}{4 \lambda}$,

$\left|I_{G}^{\alpha} f_{2}(\operatorname{ch} x)\right| \lesssim \int_{r}^{\infty} A_{c h t}^{\lambda}\left|f_{2}(\operatorname{ch} x)\right| \frac{s h^{2 \lambda} t}{(\operatorname{cht} t)^{2 \lambda+1}} d t$

$\lesssim \int_{r}^{\infty} A_{c h t}^{\lambda}\left|f_{2}(\operatorname{ch} x)\right| \frac{s h^{2 \lambda} t}{\operatorname{ch}^{\alpha} t} d t$

$\lesssim \frac{1}{\left(\operatorname{sh} \frac{r}{2}\right)^{\alpha-4 \lambda}} \int_{r}^{\infty} A_{c h t}^{\lambda}\left|f_{2}(\operatorname{ch} x)\right|\left(\frac{s h \frac{r}{2}}{s h \frac{t+r}{2}}\right)^{\alpha-4 \lambda} \operatorname{sh}^{2 \lambda} t d t$

$\lesssim \frac{1}{\left(\operatorname{sh} \frac{r}{2}\right)^{\alpha-4 \lambda}} \int_{r}^{\infty} A_{c h t}^{\lambda}\left|f_{2}(\operatorname{ch} x)\right|\left(M_{G} \chi_{H}(\operatorname{cht} t)\right)^{\frac{\alpha-4 \lambda}{4 \lambda}} \operatorname{sh}^{2 \lambda} t d t$

$\lesssim \frac{1}{\left(\operatorname{sh} \frac{r}{2}\right)^{\alpha-4 \lambda}} \int_{r}^{\infty} A_{c h t}^{\lambda}\left|f_{2}(\operatorname{ch} x)\right|\left(M_{G} \chi_{H}(\operatorname{cht} t)\right)^{\frac{\delta}{p}}\left(M_{G} \chi_{H}(\operatorname{cht} t)^{\frac{\alpha-4 \lambda}{4 \lambda}-\frac{\delta}{p}} s h^{2 \lambda} t d t\right.$.

By Hölder's inequality we have

$$
\begin{aligned}
\left|I_{G}^{\alpha} f_{2}(\operatorname{ch} x)\right| & \lesssim \frac{1}{\left(\operatorname{sh} \frac{r}{2}\right)^{\alpha-4 \lambda}}\left(\int_{r}^{\infty} A_{c h t}^{\lambda}\left|f_{2}(\operatorname{ch} x)\right|^{p}\left(M_{G} \chi_{H}(\operatorname{cht} t)\right)^{\delta} s h^{2 \lambda} t d t\right)^{\frac{1}{p}} \\
& \times\left(\int_{r}^{\infty}\left(M_{G} \chi_{H}(\operatorname{cht} t)^{\left(\frac{\alpha-4 \lambda}{4 \lambda}-\frac{\delta}{p}\right) \frac{p-1}{p}} \operatorname{sh}^{2 \lambda} t d t\right)^{\frac{p-1}{p}}\right.
\end{aligned}
$$




$$
\begin{aligned}
& =\frac{\left(\operatorname{sh} \frac{r}{2}\right)^{2 \lambda+1-\alpha}}{\left(\operatorname{sh} \frac{r}{2}\right)^{2 \lambda+1-\alpha}\left(\operatorname{sh} \frac{r}{2}\right)^{\alpha-4 \lambda}}\left(\int_{r}^{\infty} A_{c h t}^{\lambda}\left|f_{2}(\operatorname{ch} x)\right|\left(M_{G} \chi_{H}(\operatorname{cht} t)^{\delta} \operatorname{sh}^{2 \lambda} t d t\right)^{\frac{1}{p}}\right. \\
& \times\left(\int_{r}^{\infty}\left(\frac{s h \frac{r}{2}}{s h \frac{t+r}{2}}\right)^{\frac{(\alpha-4 \lambda) p-4 \lambda \delta}{p-1}} s h^{2 \lambda} t d t\right)^{\frac{p-1}{p}} \\
& \lesssim \frac{\left(\operatorname{sh} \frac{r}{2}\right)^{6 \lambda+1-2 \alpha}}{|H(0, r)|_{\lambda}^{\frac{1}{q}+1-\frac{1}{p}}}\left(\int_{r}^{\infty} A_{c h t}^{\lambda}\left|f_{2}(\operatorname{ch} x)\right|^{p}\left(M_{G} \chi_{H}(\operatorname{cht} t)\right)^{\delta} s h^{2 \lambda} t d t\right)^{\frac{1}{p}} \\
& \times\left(\int_{r}^{\infty}\left(\frac{s h \frac{r}{2}}{s h \frac{t+r}{2}}\right)^{\frac{(\alpha-4 \lambda) p-4 \lambda \delta}{p-1}} s h^{2 \lambda} t d t\right)^{\frac{p-1}{p}} \\
& \lesssim \frac{1}{|H(0, r)|_{\lambda}^{\frac{1}{q}}}\left(\int_{r}^{\infty} A_{c h t}^{\lambda}\left|f_{2}(\operatorname{ch} x)\right|\left(M_{G} \chi_{H}(\operatorname{cht} t)\right)^{\delta} s h^{2 \lambda} t d t\right)^{\frac{1}{p}} \\
& \times\left(\operatorname{sh} \frac{r}{2}\right)^{2 \lambda+1-\alpha}\left(\frac{1}{\left(\operatorname{sh} \frac{r}{2}\right)^{2 \lambda+1}} \int_{r}^{\infty} \frac{\operatorname{sh}^{2 \lambda} \frac{t}{2} \operatorname{ch}^{2 \lambda} \frac{t}{2}}{\left(\operatorname{sh} \frac{t}{2}\right)^{\frac{(\alpha-4 \lambda) p-4 \lambda \delta}{p-1}}} d t\right)^{\frac{p-1}{p}} .
\end{aligned}
$$

Further

$$
\begin{aligned}
& \frac{1}{\left(\operatorname{sh} \frac{r}{2}\right)^{2 \lambda+1}} \int_{r}^{\infty} \frac{\operatorname{sh}^{2 \lambda} \frac{t}{2} \operatorname{ch}^{2 \lambda} \frac{t}{2}}{\left(s h \frac{t}{2}\right)^{\frac{(\alpha-4 \lambda) p-4 \lambda \delta}{p-1}}} d t \\
& \leq \frac{1}{\left(\operatorname{sh} \frac{r}{2}\right)^{2 \lambda+1}} \int_{r}^{\infty} \frac{d\left(s h \frac{t}{2}\right)}{\left(\operatorname{sh} \frac{t}{2}\right)^{\frac{(\alpha-4 \lambda) p-4 \lambda \delta}{p-1}-4 \lambda+1}} \\
& \lesssim \frac{1}{\left(\operatorname{sh} \frac{r}{2}\right)^{2 \lambda+1}} \cdot \frac{1}{\left(\operatorname{sh} \frac{r}{2}\right)^{\frac{(\alpha-4 \lambda) p-4 \lambda \delta-4 \lambda(p-1)}{p-1}}} \\
& \lesssim \frac{1}{\left(\operatorname{sh} \frac{r}{2}\right)^{\frac{(\alpha-8 \lambda) p+4 \lambda-4 \lambda \delta+(2 \lambda+1)(p-1)}{p-1}}}=\frac{1}{\left(\operatorname{sh} \frac{r}{2}\right)^{\frac{(\alpha-6 \lambda+1) p-4 \lambda \delta+2 \lambda-1}{p-1}}} .
\end{aligned}
$$

From this and (5.8) we obtain

$$
\begin{aligned}
& \left(\operatorname{sh} \frac{r}{2}\right)^{2 \lambda+1-\alpha}\left(\frac{1}{\left(\operatorname{sh} \frac{r}{2}\right)^{2 \lambda+1}} \int_{r}^{\infty} \frac{\operatorname{sh}^{2 \lambda} \frac{t}{2} \operatorname{ch}^{2 \lambda} \frac{t}{2}}{\left(\operatorname{sh} \frac{t}{2}\right)^{\frac{((-4 \lambda) p-4 \lambda \delta}{p-1}}} d t\right)^{\frac{p-1}{p}} \\
& \lesssim \frac{1}{\left(\operatorname{sh} \frac{r}{2}\right)^{\frac{(\alpha-6 \lambda+1) p-4 \lambda \delta+2 \lambda-1-(2 \lambda+1-\alpha) p}{p}}}
\end{aligned}
$$

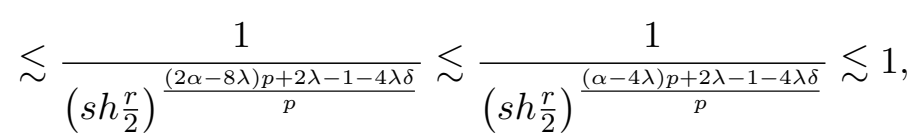

From this and (5.8), we have

$$
\left|I_{G}^{\alpha} f_{2}(\operatorname{ch} x)\right| \lesssim|H(0, r)|_{\lambda}^{-\frac{1}{q}}\left(\int_{r}^{\infty} A_{c h t}^{\lambda}\left|f_{2} \operatorname{ch} x\right|^{p}\left(M_{G} \chi_{H}(\operatorname{cht} t)\right)^{\delta} s h^{2 \lambda} t d t\right)^{\frac{1}{p}} .
$$


Combining(5.5), (5.7) and (5.9), by Lemma 4.4 we obtain

$$
\begin{aligned}
\left|I_{G}^{\alpha} f_{2}(\operatorname{ch} x)\right| & \lesssim|H(0, r)|_{\lambda}^{-\frac{1}{q}}\left(\int_{r}^{\infty} A_{c h t}^{\lambda}|f c h x|^{p}\left(M_{G} \chi_{H}(c h t)\right)^{\delta} s h^{2 \lambda} t d t\right)^{\frac{1}{p}} \\
& \lesssim|H(0, r)|_{\lambda}^{-\frac{1}{q}} w(r)^{\frac{1}{p}}\|f\|_{M_{p, \lambda, w}}, \text { for } x \in H(0, r)
\end{aligned}
$$

and

$$
\left\{w(r)^{-\frac{q}{p}} \int_{H(0, r)}\left|I_{G}^{\alpha} f_{2}(\operatorname{ch} x)\right|^{q} s h^{2 \lambda} x d x\right\}^{\frac{1}{q}} \lesssim\|f\|_{M_{p, \lambda, w}} .
$$

By (5.1) and (5.10) we get (3.5).

(ii) For $f \in L_{1, \lambda, w}\left(\mathbb{R}_{+}, G\right)$ and for $f \in H(0, r)$ let $f=f_{1}+f_{2}, f_{1}=f \chi_{H}$. By Theorem $5.1, I_{G}^{\alpha}$ is bounded from $L_{1, \lambda}\left(\mathbb{R}_{+}, G\right)$ to $W L_{q, \lambda}\left(\mathbb{R}_{+}, G\right)$

$$
\left|\left\{x \in H(0, r):\left|I_{G}^{\alpha} f_{1}(\operatorname{ch} x)\right|>\beta\right\}\right|_{\lambda} \lesssim\left(\frac{1}{\beta}\left\|f_{1}\right\|_{L_{1, \lambda}}\right)^{q} \lesssim\left(\frac{w(r)}{\beta}\|f\|_{M_{1, \lambda, w}}\right)^{q} .
$$

It follows from (5.2) and Lemma 4.4 with $p=1, \delta=1-\frac{\alpha}{2 \lambda+1}=\frac{1}{q}$ that at $0<r<2$

$$
\begin{aligned}
\left|I_{G}^{\alpha} f_{2}(\operatorname{ch} x)\right| & \lesssim|H(0, r)|_{\lambda}^{-\frac{1}{q}} \int_{r}^{\infty} A_{c h t}^{\lambda}\left|f_{2}(\operatorname{ch} x)\right|^{p}\left(M_{G} \chi_{H}(c h t)\right)^{\frac{1}{q}} s h^{2 \lambda} t d t \\
& \lesssim|H(0, r)|_{\lambda}^{-\frac{1}{q}} w(r)\|f\|_{M_{1, \lambda, w}} \text { for } x \in H(0, r) .
\end{aligned}
$$

And suppose $\delta=1-\frac{\alpha}{4 \lambda}=\frac{1}{q}$ at $2 \leq r<\infty$

$$
\left|I_{G}^{\alpha} f_{2}(\operatorname{ch} x)\right| \lesssim|H(0, r)|_{\lambda}^{-\frac{1}{q}} w(r)\|f\|_{M_{1, \lambda, w}}, \text { for } x \in H(0, r) .
$$

From (5.12) and (5.13) we have

$$
\begin{aligned}
|\{x \in H(0, r)\}:| I_{G}^{\alpha} f_{2}(\operatorname{ch} x)|>\beta|_{\lambda} & \lesssim \int_{H(0, r)}\left(\frac{A_{c h t}^{\lambda}\left|I_{G}^{\alpha} f_{2}(\operatorname{ch} x)\right|}{\beta}\right)^{q} s h^{2 \lambda} t d t \\
& \lesssim\left(\frac{w(r)}{\beta}\|f\|_{M_{1, \lambda, w}}\right)^{q} .
\end{aligned}
$$

Combining (5.11) and (5.14) we obtain (3.6).

Acknowledgements. The first author was partially supported by the grant of 1st Azerbaijan-Russia Joint Grant Competition (Grant No. EIF-BGM-4-RFTF$1 / 2017-21 / 01 / 1)$. The authors thank to the referee for the constructive comments and recommendations, which definitely help to improve the readability and quality of the paper.

\section{References}

[1] L. Durant, P. M.Fisbane, and L. M. Simmons, Expansion formulas and addition theorems for Gegenbauer functions, J.Math. Phys. 17 (1976), 1933-1948.

[2] C. Fefferman, E.M. Stein, Some maximal inequalities, Amer. J. Math. 93 (1971), 107-115.

[3] V.S. Guliyev, E.J. Ibrahimov, Necessary and sufficient condition for the boundedness of the Gegenbauer-Riesz potential on Morrey Spaces, Georgian Math. J. (2018), 1-13.

[4] V.S. Guliyev, E. Ibrahimov, Necessary and sufficient conditions for the boundedness of the Gegenbauer potential, Mat. Zametki 105 (2019), 681-691. 
[5] V.S. Guliyev, E. Ibrahimov, Necessary and sufficient conditions for the boundedness of the Gegenbauer-Riesz potential in modified Morrey spaces, Trans. A. Razmadze Math. Inst. 170 (2019), 1-15.

[6] V.S. Guliyev, E.J. Ibrahimov, S.Ar. Jafarova, Gegenbauer Transformation, NikolskiBesov Spaces Generalized by Gegenbauer operator and Their Approximation Characteristics, Advances in Analysis 2 (2017), 167-194.

[7] V.S. Guliyev, E.J. Ibrahimov, On equivalent normalizations of functional spaces associated with the generalized Gegenbauer shift, Anal. Math 34 (2008), 83-103 (Russian).

[8] V.S. Guliyev, E.J. Ibrahimov, Generalized Gegenbauer shift and some problems of the theory of approximation of functions on the metric of $L_{2, \lambda}$, Trans. Natl. Acad. Sci. Azerb. Ser. Phys.-Tech. Math. Sci. Mathematics 35 (2015), 19-51.

[9] E.J.Ibrahimov, A. Akbulut, The Hardy-Littlewood-Sobolev theorem for Riesz potential generated by Gegenbauer operator, Trans.of A.Razmadze Math.Init. 170 (2016), 166-199.

[10] E.J. Ibrahimov, S.Ar.Jafarova, On boundedness of the Riesz potential generated by Gegenbauer differential on Morrey spaces, Trans. Natl. Acad. Sci. Azerb. Ser. Phys.-Tech. Math. Sci. Mathematics 37 (2017), 49-70.

[11] E.J. Ibrahimov, On Gegenbauer transformation on the half-line, Georgian Math. J. 18 (2011), 497-515.

[12] V. Kokilashvili, S. Samko , Singular integrals in Weighted Lebesgue Spaces with Variable Exponent, Georgian Math. J. 10 (2003), 145-156.

[13] B.M. Levitan, Expansion in Fourier series and integrals with Bessel functions, Uspekhi Mat. Nauk 6 (1951), 102-143.

[14] B.M. Levitan, Theory of Generalized Shift Operators, [in Russian] Nauka, Moscow, 1973.

[15] Lu Sh., Ding Y, Yan D, Singular integrals and related topics, World Scientific Publishing, 2007.

[16] E. Nakai, Hardy-Littlewood Maximal operator, Singular Integral Operators and the Riesz Potentials on Generalized Morrey Spaces, Math. Nachr. 166 (1994),95-103.

Elman J. Ibrahimov

Institute of Mathematics and Mechanics of NAS of Azerbaijan, Baku, Azerbaijan

E-mail address: elmanibrahimov@yahoo.com

Saadat A. Jafarova

Azerbaijan State Economic University, AZ1001, Baku, Azerbaijan

E-mail address: sada-jafarova@rambler.ru

S. Elifnur Ekincioglu

Department of Mathematics, Dumlupinar University, 43100 Kutahya, Turkey

E-mail address: elifnurekincioglu@gmail.com

Received: June 17, 2019; Revised: December 22, 2019; Accepted: March 11, 2020 